\title{
Relativistic Paradox of a Uniformly Charged Sphere Moving with Constant Velocity
}

\author{
Vladimir Alexander Leus \\ Department of Electrical Engineering and Electronics, University of Liverpool, Liverpool, UK \\ Email: v.leus@liverpool.ac.uk
}

How to cite this paper: Leus, V.A. (2020) Relativistic Paradox of a Uniformly Charged Sphere Moving with Constant Velocity. Journal of Modern Physics, 11, 145-155. https://doi.org/10.4236/jmp.2020.111009

Received: November 11, 2019

Accepted: January 14, 2020

Published: January 17, 2020

Copyright $\odot 2020$ by author(s) and Scientific Research Publishing Inc. This work is licensed under the Creative Commons Attribution International License (CC BY 4.0).

http://creativecommons.org/licenses/by/4.0/

\begin{abstract}
A magneto-electric field appearing in a laboratory due to moving charges has unusual properties. In particular, such a field of kinematical origin does not obey the wave equation with a non-relativistic velocity instead of light speed; so, its movement resembles that of a rigid body. In this paper the field of a uniformly charged sphere moving at constant velocity is considered. Relativistic axiom, implicitly used in the derivation of formulas describing a kinematic deformation for the proper spherical field from the point of view of a fixed observer, is revealed. A discrepancy was found between the generally accepted idea of the configuration of a deformed field and its real geometry. It is shown that the correct interpretation of known formulas leads to a logical contradiction, which cannot be eliminated within the framework of the theory of relativity. A scheme of a decisive experiment is proposed.
\end{abstract}

\section{Keywords}

Charged Sphere, Uniform Motion, Deformed Field, Implicit Axiom, Relativistic Paradox

\section{Introduction}

The Coulomb field of a stationary point charge, which has the simplest spherical symmetry with a strength decreasing inversely proportional to the square of the distance from the source, undergoes a very exotic deformation, if a charge (or an observer) is forced to enter a state of uniform rectilinear motion. It was first pointed out by Oliver Heaviside [1].

The chronological continuity between the relativistic concept of electromagnetism and Maxwell's theory is well known. It was clearly manifested in the study of the motion of a point charge, whose field, from the point of view of a stationary observer in the laboratory, loses its Coulomb configuration. In this 
case, the vector field of electric strength $\boldsymbol{E}$ satisfies the wave equation

$$
\nabla^{2} \boldsymbol{E}-\frac{1}{c^{2}} \frac{\partial^{2} \boldsymbol{E}}{\partial t^{2}}=\mathbf{0} \text {. }
$$

As a parameter, it includes the speed of light $c$. The accompanying magneto-kinematic field of vectors $\boldsymbol{H}$ is subject to the same equation

$$
\nabla^{2} \boldsymbol{H}-\frac{1}{c^{2}} \frac{\partial^{2} \boldsymbol{H}}{\partial t^{2}}=\mathbf{0} .
$$

Consequently, the displacement of a field in the laboratory space with a velocity $\boldsymbol{v}$ has a non-wave nature; rather, it is akin to the motion of a rigid body for the non-relativistic case [2] and for the relativistic one [3] as well.

The following demonstrates the fact that in deriving formulas describing the kinematic deformation of a field for a stationary observer, a special relativistic axiom is implicitly applied. At the same time, a discrepancy between the generally accepted idea of the configuration of a deformed field with respect to its real geometry is revealed. The field of a uniformly charged sphere moving at a constant speed is considered. It is shown that the correct interpretation of known formulae leads to a logical contradiction, which cannot be eliminated within the framework of the theory of relativity. A scheme of the experiment is proposed which is to confirm or refute the generally accepted formula for a deformed electrostatic field of a charge moving at a constant velocity.

\section{Relativistic Derivation of the Expression for the Field of a Moving Charge, Based on a Hidden Axiom}

Recall how the expressions for the fields of a moving point charge are derived. Using the four-dimensional formalism of the special theory of relativity (STR), the authors of the book "Field Theory" [4] come to expressions of the scalar and vector potentials, derived by Lienard and Wiechert long before the birth of the STR. Using then Maxwell's electromagnetic theory, in the end, a general formula is obtained for the electric and magnetic field vectors of a single point charge making a given motion along a path $\boldsymbol{r}=\boldsymbol{r}_{0}(t)$ (Figure 1 ).

"According to the formulas for retarded potentials, the field at the point of observation $P(x, y, z)$ at time $t$ is determined by the state of motion of the charge at the earlier time $t^{\prime}$, for which the time of propagation of the light signal from the point $\boldsymbol{r}_{e}\left(t^{\prime}\right)$, where the charge was located, to the field point $P$ just

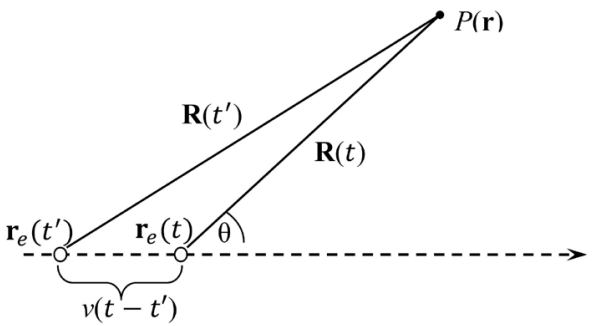

Figure 1. A point charge moving along a given path. 
coincides with the difference $t-t^{\prime}$. Let $\boldsymbol{R}(t)=\boldsymbol{r}-\boldsymbol{r}_{e}(t)$ be the radius vector from the charge e to the point $P$; like $r_{e}(t)$ it is a given function of the time. Then the time $t^{\prime}$ is determined by the equation

$$
\frac{t^{\prime}+R\left(t^{\prime}\right)}{c}=t .
$$

For each value of this equation has just one root $t^{\prime}$ ([4], p. 212). This is how $\$ 63$ "Lienard-Wiechert potentials" begins in the book by Landau and Lifshitz.

"Passing now again to three-dimensional notation, we obtain the following expressions for the field potentials of an arbitrarily moving point charge:

$$
\varphi=\frac{e}{\left(R-\frac{\boldsymbol{v} \cdot \boldsymbol{R}}{c}\right)}, \quad \boldsymbol{A}=\frac{e \boldsymbol{v}}{c\left(R-\frac{\boldsymbol{v} \cdot \boldsymbol{R}}{c}\right)},
$$

where $\boldsymbol{R}$ is the radius vector, taken from the point where the charge is located to the point of observation $P$, and all the quantities on the right sides of the equations must be evaluated at the time $t^{\prime}$ determined from the previous equation. The potentials of the field, in the form $\left.{ }^{(*}\right)$, are called the Lienard-Wiechert potentials" ([4], p. 213).

Let us turn to the case of uniform and rectilinear motion of a point charge along the trajectory $\boldsymbol{r}=\boldsymbol{r}_{0}(t)$ with a constant velocity $\boldsymbol{v}$. We introduce the notation $\boldsymbol{R}^{\prime}$ and $\boldsymbol{R}$ for the vectors $\boldsymbol{R}\left(t^{\prime}\right)$ and $\boldsymbol{R}(t)$, respectively (Figure 1). Maxwell formulas are applied to obtain the strengths of the electric and magnetic fields:

$$
\boldsymbol{E}=-\frac{1}{c} \frac{\partial \boldsymbol{A}}{\partial t}-\operatorname{grad} \varphi, \boldsymbol{H}=\operatorname{rot} \boldsymbol{A},
$$

which yields the expressions

$$
\boldsymbol{E}=e \frac{1-v^{2} / c^{2}}{\left(R^{\prime}-\frac{\boldsymbol{R}^{\prime} \boldsymbol{v}}{c}\right)^{3}}\left(\boldsymbol{R}^{\prime}-\frac{\boldsymbol{v}}{c} R^{\prime}\right) ; \quad \boldsymbol{H}=\frac{1}{R^{\prime}}\left[\boldsymbol{R}^{\prime} \boldsymbol{E}\right] .
$$

Here, the symbol [..] denotes vector product.

"Indeed, at constant speed, the difference

$$
\boldsymbol{R}^{\prime}-\frac{\boldsymbol{v}}{C} R^{\prime}=\boldsymbol{R}^{\prime}-\boldsymbol{v}\left(t-t^{\prime}\right)
$$

there is a vector $\boldsymbol{R}$ from charge to observation point at the very moment of observation. It is also easy to verify by checking directly that

$$
R^{\prime}-\frac{1}{C} \boldsymbol{R}^{\prime} \boldsymbol{v}=\sqrt{R^{2}-\frac{1}{c^{2}}[\boldsymbol{v} \boldsymbol{R}]^{2}}=R \sqrt{1-\frac{v^{2}}{c^{2}} \sin \theta_{t}},
$$

where $\theta_{t}$ is the angle between $\boldsymbol{R}$ and $\boldsymbol{v}$ "([4], p. 215).

Now you can substitute the right side of the Formula $\left(^{* * *}\right)$ in the numerator, and the right side of the Formula $\left({ }^{* * *}\right)$ in the denominator of the first term in the expression (2): 


$$
\boldsymbol{E}(\theta)=e \frac{\left(1-v^{2} / c^{2}\right) \boldsymbol{R}}{R^{3}\left(1-\left(v^{2} / c^{2}\right) \sin ^{2} \theta\right)^{3 / 2}} .
$$

We have arrived at a formula that describes the distribution in space of the electric intensity in a field of a point charge, which moves relative to the observer along a straight line at a constant speed. The authors of the book [4] use the CGSE system of units, while the entry in the international system of units (SI) is

$$
\boldsymbol{E}=\frac{q\left(1-v^{2} / c^{2}\right)}{4 \pi \varepsilon_{0} r^{3}\left(1-\left(v^{2} / c^{2}\right) \sin ^{2} \theta\right)^{3 / 2}} \boldsymbol{r},
$$

where the designation of the individual charge of an electron is replaced by the universal letter $q$.

As you can see, in the SRT there is complete continuity in what is concerning the terminology of retarded potentials. This way of reasoning leaves behind the scene an arbitrary assumption of an incessant outflow of a field from its source, the charge. In fact, since the potential propagates from the field source at the speed of light " $c$ " regardless of the kind of movement the charge performs, this outflow also takes place when the charge moves with a constant velocity $\boldsymbol{v}$. And since the speed of light does not depend on $v$, the outflow of potential from the charge remains unchanged when the speed $\boldsymbol{v}$ tends to zero. Therefore, we have to agree that an unceasing extrovert flow from a charged source exists in any electrostatic field. This statement should be explicitly introduced into the axiomatic basis, when the SRT is transferred to the realm of electromagnetism.

\section{Relativistic Paradox}

The expression (3) can be obtained within the framework of a pure STR, without resorting to the Lienard-Wiechert potentials. Section 20 of the textbook [5], called "Moving charge field", Part II, entitled "Theory of Relativity", deals with the Coulomb field of a point charge deformed to a configuration (3) for an observer moving at a constant velocity $-\boldsymbol{v}$ along with respect to charge. Of course, with the same success, we can speak of the charge moving at a velocity $v$ relative to the observer. "In the reference frame K, moving along with the charge, there is no magnetic field, and the electric field potential is expressed by the formula $\varphi^{\prime}=e / r^{\prime}$." ([5], p. 254). The following conclusion is based on the relativistic formula for the transformation of the scalar potential:

$$
\varphi=\frac{\varphi^{\prime}+\frac{v}{c} A_{x}^{\prime}}{\sqrt{1-\frac{v^{2}}{c^{2}}}} .
$$

On page 256 we read: "The scalar potential $\varphi$ has a constant value on the surface of the ellipsoid 


$$
(x-v t)^{2}+\left(1-\frac{v^{2}}{c^{2}}\right)\left(y^{2}+z^{2}\right)=\text { const } .
$$

This ellipsoid is obtained from the sphere by compressing it in the direction of the $x$-axis into $1: \sqrt{1-\frac{v^{2}}{c^{2}}}$ times". We apply the formalism used there to the field of the conducting sphere of unit radius, over which the charge $q$ is uniformly distributed.

This sphere has a centre at the origin $\left(x^{\prime}, y^{\prime}, z^{\prime}\right)$ of a primed IRF' (Inertial Reference Frame), moving in a straight line with a constant speed $v=(v, 0,0)$ relative to the non-primed (laboratory) IRF with coordinate axes $(0 x, 0 y, 0 z)$ parallel to corresponding axes of the primed coordinate system. For the origin of time, the moment is taken when the origins of spatial coordinate systems coincide; therefore, the position of the centre of the sphere in the non-primed IRF is the point with coordinates $x=v t, y=0, z=0$. The scalar potential outside the sphere in its own IRF' coincides with the Coulomb potential (in this section, formulas are written in the Gaussian system of units)

$$
\varphi^{\prime}=\frac{q}{r^{\prime}}
$$

On the sphere itself and inside it, it has a constant value $\varphi_{0}^{\prime}=q / 1$. According to the Lorentz transformations for electromagnetic fields, the scalar potential in a non-primed system is

$$
\varphi=\gamma \varphi^{\prime}=\gamma \frac{q}{r^{\prime}}
$$

since the vector potential here is zero. The Lorentz transformations for coordinates give the expression of the primed radius-vector from the point of view of the non-primed IRF in the form $r^{\prime}=\sqrt{\gamma^{2}(x-v t)^{2}+y^{2}+z^{2}}$. Substitute this expression in (4):

$$
\varphi=\gamma \frac{q}{\sqrt{\gamma^{2}(x-v t)^{2}+y^{2}+z^{2}}}=\frac{q}{\sqrt{(x-v t)^{2}+\frac{1}{\gamma^{2}}\left(y^{2}+z^{2}\right)}} .
$$

Hence, the surface of level $\varphi=a=$ const is described by the equation

$$
(x-v t)^{2}+\frac{1}{\gamma^{2}}\left(y^{2}+z^{2}\right)=\frac{q^{2}}{a^{2}} .
$$

Here it is logical to investigate the question on the deformation of the charge carrier-the conducting sphere of a unit radius-upon transition to a non-primed IRF. From the point of view of the non-primed IRF, the carrier of the moving charge loses its spherical shape. Consider the section of the sphere by the plane $z=0$ (Figure 2). Let at the moment $t^{\prime}$ of the own time in the primed IRF' the $x^{\prime}$-coordinates at the ends of the chord, parallel to the $x$-axis, be measured. We have two events $\left(x_{1}^{\prime}, y^{\prime}, t^{\prime}\right)$ and $\left(x_{2}^{\prime}, y^{\prime}, t^{\prime}\right)$, where $y^{\prime}$ is the ordinate of the chord. We translate them by the transformations of Lorentz into a non-primed IRF: 


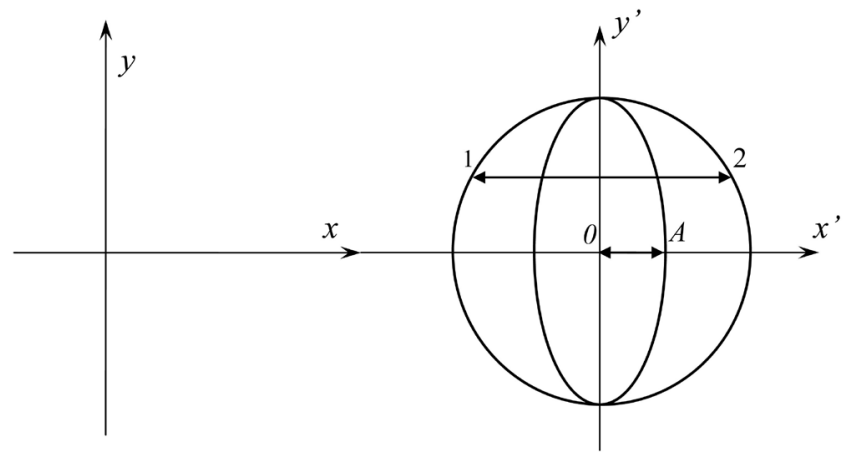

Figure 2. Deformation of the surface of a charged sphere.

$$
\begin{aligned}
& x_{1}=\gamma\left(x_{1}^{\prime}+v t^{\prime}\right), y_{1}=y^{\prime}, t_{1}=\gamma\left(t^{\prime}+\frac{v}{c^{2}} x_{1}^{\prime}\right) \\
& x_{2}=\gamma\left(x_{2}^{\prime}+v t^{\prime}\right), y_{2}=y^{\prime}, t_{2}=\gamma\left(t^{\prime}+\frac{v}{c^{2}} x_{2}^{\prime}\right) .
\end{aligned}
$$

For the time interval $t_{2}-t_{1}=\gamma\left(\frac{v}{c^{2}} x_{2}^{\prime}-\frac{v}{c^{2}} x_{1}^{\prime}\right)=\gamma \frac{v}{c^{2}}\left(x_{2}^{\prime}-x_{1}^{\prime}\right)$ the second end of the chord managed to drive off at a speed $v$ to a distance $\gamma \frac{v^{2}}{c^{2}}\left(x_{2}^{\prime}-x_{1}^{\prime}\right)$, which should be subtracted from the abscissa difference $x_{2}-x_{1}$ to get chord length in non-primed IRF:

$$
\begin{aligned}
l & =\left(x_{2}-x_{1}\right)-\gamma \frac{v^{2}}{c^{2}}\left(x_{2}^{\prime}-x_{1}^{\prime}\right) \\
& =\gamma\left(x_{2}^{\prime}-x_{1}^{\prime}\right)-\gamma \frac{v^{2}}{c^{2}}\left(x_{2}^{\prime}-x_{1}^{\prime}\right) \\
& =\gamma\left(1-\frac{v^{2}}{c^{2}}\right)\left(x_{2}^{\prime}-x_{1}^{\prime}\right)=\frac{l^{\prime}}{\gamma}
\end{aligned}
$$

By virtue of the arbitrariness in the choice of the chord and in the choice of the section by the plane passing through the abscissa axis, we conclude that the entire sphere undergoes longitudinal compression by $\gamma$ times and turns into an ellipsoid of rotation. The half-axis $0 \mathrm{~A}$ turns out to be $\gamma$ times shorter than the radius of the sphere, that is, its length is $1 / \gamma$. Just the same deformation is mentioned in the cited above excerpt from the textbook [5] with respect to the equipotential surface (a surface of the potential equivalency).

On the unit sphere itself, the potential is $\varphi_{0}^{\prime}=q / 1$, and this spherical surface of level $a=q / 1$ becomes, according to [5], an ellipsoid with the equation

$$
(x-v t)^{2}+\frac{1}{\gamma^{2}}\left(y^{2}+z^{2}\right)=1
$$

in terms of the non-primed IRF. If you believe the textbook's statement [5] that "This ellipsoid is obtained from a sphere by compressing it along the $x$-axis by $1: \sqrt{1-\frac{v^{2}}{c^{2}}}:$ times", then there is nothing better to desire. Indeed, the quantity 
$1: \sqrt{1-\frac{v^{2}}{c^{2}}}=$ times and, as we found out, namely by $\gamma$ times that the sphere-a carrier of charge is compressed. Thus, the potential of a charged sphere promises to be an invariant of Lorentz transformations. But, alas, it appears to be too good to be true. The ideal is "only a dream" for us, and the desired harmony does not withstand the "checking up with algebra"!

To put it mildly, strange misunderstandings are associated with the field configuration of a uniformly moving charge. For example, on the behaviour of vectors $E$ we read in ([4], p. 126): "It can be said visually about the electric field of a moving charge as though it is "compressed in the direction of motion". In fact, the deformation also contains transverse dilatation, and the lines of the constant module $E=$ const have a rather complicated guitar-like shape (see [6]).

As for the picture of the equipotential surfaces, we are faced with fata morgana, which for decades has been unconditionally accepted by all readers for an objective reality ("When there is no real life, they live in mirages. Still, better than nothing." A.P. Chekhov). The fact is that the notorious equation in the quotation from ([5], p. 256) pertains to the ellipsoid, which is obtained from the sphere when it is stretched in directions orthogonal to the $x$-axis by $\gamma$ times. The elimination of the traditional ghost has fatal consequences for the relativistic interpretation of the field of a uniformly moving charge. When transforming to IRF, the equipotential surface of the charged sphere is converted not into a sphere compressed to an ellipsoid, but into an ellipsoid stretched across the velocity. Whereas into the ellipsoid-the charge carrier-the sphere of radius $r^{\prime}=1$ is converted so that this ellipsoid proves to be situated in the internal cavity of the equipotential ellipsoid.

In Figure 3 the section by the plane $z=0$ is present where two ellipsoids are shown. One is being a result of the longitudinal compression of the "unit" sphere by a factor of $\gamma$, and another is being a result of the transverse dilatation of this sphere by a factor of $\gamma$. The first of them is a charge carrier such as it exists in the IRF where it is described by the equation

$$
\gamma^{2}(x-v t)^{2}+y^{2}+z^{2}=1
$$

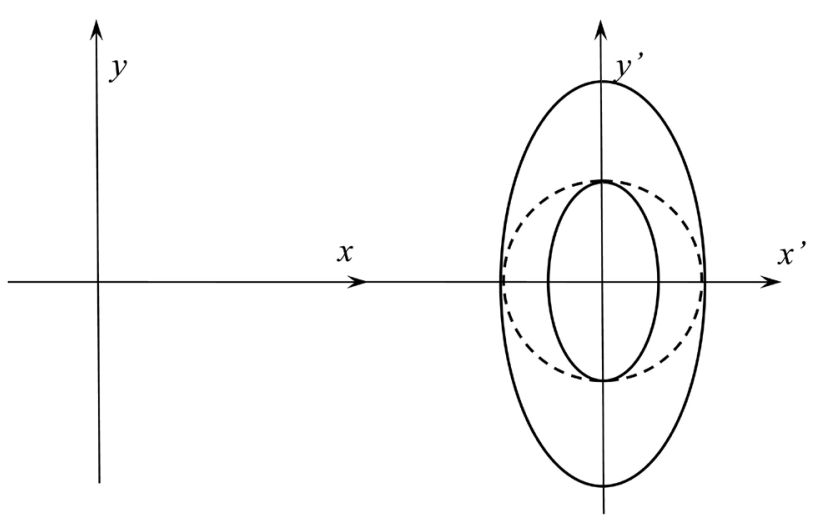

Figure 3. Separation of equipotential surface from charged ellipsoid. 
The second is the equipotential surface $\varphi_{0}=q / 1$ of the electrostatic field, objectively existing in the same IRF, with Equation (6). The second ellipsoid encloses the first, and between them is a layer of variable thickness. In the longitudinal direction, the layer thickness is $(\gamma-1) / \gamma$, and in the transverse direction, the thickness is $(\gamma-1)$, so that with increasing speed $\boldsymbol{v}$, the longitudinal thickness approaches unity, while the transverse one increases without limit.

What lies within this layer? In accordance with the relativistic tradition, an ellipsoidal charge carrier retains a spherical potential, and in the layer under consideration everywhere we have

$$
\boldsymbol{E}=-\frac{1}{c} \frac{\partial \boldsymbol{A}}{\partial t}-\operatorname{grad} \varphi=\mathbf{0},
$$

because the scalar potential $\varphi=q / 1=$ const in a finite region, on the boundary of which it is constant, and the vector potential $\boldsymbol{A}=\varphi \boldsymbol{v}$, as a result, is also constant in this region and the partial derivative with respect to time is zero. It turns out that the charged ellipsoidal shell is paradoxically immersed in a volume free of the electrostatic field, and only from the outer boundary of this volume does the space, penetrated by the field (3) with equipotential surfaces of type (5), is starting.

The Ostrogradsky-Gauss theorem does not work in the resulting bizarre electrostatic field. This becomes apparent when the test closed surface surrounding the charge carrier is selected entirely inside the neutral layer. The flux of vector $\boldsymbol{E}$ through this surface is zero, while the integral charge inside is non-zero.

We will try to get away from this electric monster by allowing the scalar potential $\varphi_{1}$ of the ellipsoidal charge carrier to be different from $\varphi_{0}: \varphi_{1}<q / 1$ or $\varphi_{1}>q / 1$. In this case, we are faced with another paradox. Suppose there are two identical and equally charged conductive spheres uniformly moving in the laboratory IRF to meet one another. Own IRF' of the first sphere moves with velocity $\boldsymbol{v}$ and own IRF" of the second sphere moves with velocity $(-\boldsymbol{v})$, relative to the non-primed IRF. At the zero moment of time $\left(t=t^{\prime}=t^{\prime \prime}=0\right)$, when the origins of all three IRFs coincide, the charge carriers touch each other by the top and bottom limit points respectively. From the point of view of the laboratory IRF, both ellipsoids have the same values of scalar potentials $\varphi_{1}=\varphi_{2} \neq q / 1$ and the voltage between them is zero. But in the IRF' the picture is devoid of such symmetry, because the first sphere has the potential $\varphi_{1}^{\prime}=q / 1$, whereas the second sphere, compressed into an ellipsoid, has the potential $\varphi_{2}^{\prime} \neq q / 1$. Between them there is a voltage $U=\varphi_{2}^{\prime}-\varphi_{1}^{\prime} \neq 0$, causing a discharge. An electrical discharge occurs also in IRF", but only with oppositely directed current. The pulsed current arising from the discharge becomes a source of electromagnetic wave, and its objective existence does not depend on the inertial reference frame. Consequently, the EM-wave should also appear in the laboratory IRF, where there is no current at all, that is, radiation arises from literally nothing! A sceptic who doubts the "promptness" of conduction electrons can be calmed by a metal tape longitudinally stretched in IRF, which will sufficiently prolong the contact time of charged spheres (ellipsoids). 


\section{The Possibility of Experimental Verification}

So, contrary to the generally accepted opinion of the complete compatibility of the SRT with Maxwellian electrodynamics, the field of a uniformly moving charge throws to physicists in general, and to experimenters especially, a serious challenge. Indeed, since the time of Heaviside's guess, that is, for more than 130 years, the "relativistic" configuration of such a field has not been confirmed by experience. In [6], the idea of a decisive experiment was proposed, promising to establish whether the prevailing view really corresponds to the natural state of affairs.

Suppose there is a high-ampere electron beam supported in a rectilinear vacuum tube with a length of $2 s$ ( $x$-axis in Figure 4 ). At the point $(0, h)$, the beam element $\mathrm{d} x$ generates an electric field with intensity $\mathrm{d} \boldsymbol{E}^{\prime}$, and a beam element symmetric to it generates a field with intensity $\mathrm{d} \boldsymbol{E}^{\prime \prime}$. The sum $\mathrm{d} \boldsymbol{E}^{\prime}+\mathrm{d} \boldsymbol{E}^{\prime \prime}=\mathrm{d} \boldsymbol{E}$ is directed along the $y$ axis and has an absolute value

$$
\mathrm{d} E=\frac{2 \sigma \mathrm{d} x}{4 \pi \varepsilon_{0}} \frac{\left(1-\beta^{2}\right) \sin \theta}{\left(x^{2}+h^{2}\right)\left(1-\beta^{2} \sin ^{2} \theta\right)^{\frac{3}{2}}}=\frac{\sigma\left(1-\beta^{2}\right) \mathrm{d} x}{2 \pi \varepsilon_{0}\left[x^{2}+\left(1-\beta^{2}\right) h^{2}\right]^{\frac{3}{2}}}
$$

according to Formula (3). Here the letter $\sigma$ denotes the linear charge density, $\sin \theta=h / \sqrt{x^{2}+h^{2}}$, and $\beta=v / c$. The total electrical intensity is obtained by integrating over the length of the beam:

$$
E=\int_{0}^{s} \mathrm{~d} E=\frac{\sigma\left(1-\beta^{2}\right)}{2 \pi \varepsilon_{0}} \int_{0}^{s} \frac{\mathrm{d} x}{\left[x^{2}+\left(1-\beta^{2}\right) h^{2}\right]^{\frac{3}{2}}}=\frac{\sigma}{2 \pi \varepsilon_{0}} \cdot \frac{s}{h\left[s^{2}+\left(1-\beta^{2}\right) h^{2}\right]^{\frac{1}{2}}} .
$$

Expressing the charge density through the electron velocity in the beam and the current strength in it $I=\sigma v$, we get

$$
E(h, s, \beta, I)=\frac{I s}{2 \pi \varepsilon_{0} v h \sqrt{s^{2}+\left(1-\beta^{2}\right) h^{2}}}=\frac{I s}{2 \pi \varepsilon_{0} \beta c h \sqrt{s^{2}+\left(1-\beta^{2}\right) h^{2}}} .
$$

If the electric field of a moving point charge remains Coulomb at any speed, then the dependence of $E$ on $\beta$ will be different:

$$
E_{0}(\beta)=\frac{I s}{2 \pi \varepsilon_{0} \beta c h \sqrt{s^{2}+h^{2}}} .
$$

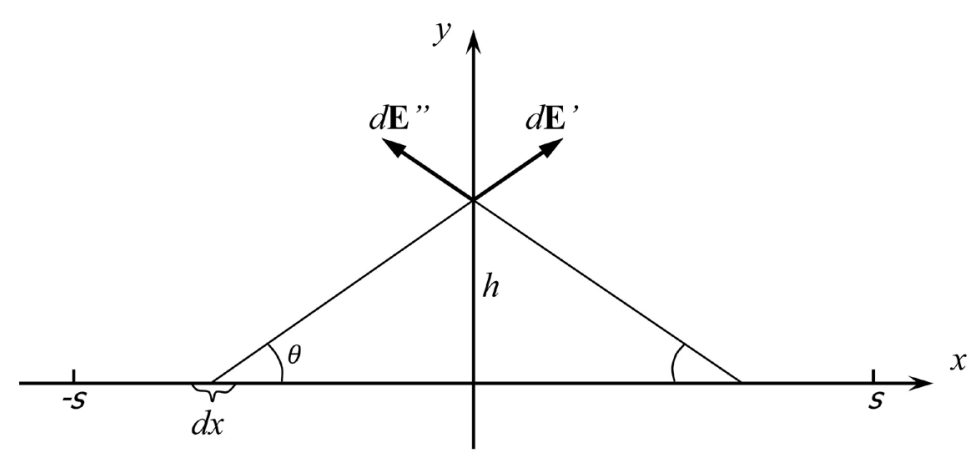

Figure 4. Experiment in a rectilinear vacuum tube. 
The well-known experiment on the detection of the electric field around a superconducting ring with current [7] is extremely difficult to perform, since it is necessary to detect the effect of the second order of smallness with respect to the ratio $v / c=\beta$. The difference between the electro-kinematic field created by the moving conduction electrons and the electrostatic field of the stationary ions of the conductor crystal lattice leads to it. The letter $v$ refers here to the drift velocity of conduction electrons, which no snail would envy. Moreover, as was shown later, the result of the experiment cannot be interpreted unambiguously: "the experiment performed by Edwards et al. is not a test of Maxwells equations in the most general case, but a test of these equations in the particular case of the superconducting state" [8].

The experimental scheme proposed here is completely free from the main obstacle facing the authors of the work [7]. At a current of one ampere at a distance of half a meter from the middle of the beam one meter in length, the electric intensities calculated by Formulas (7) and (8), respectively, are equal to $287 \mathrm{~V} / \mathrm{m}$ and $281 \mathrm{~V} / \mathrm{m}$ with $\beta=0.3$. When $\beta=0.99$, the values of $119 \mathrm{~V} / \mathrm{m}$ and 85 $\mathrm{V} / \mathrm{m}$ are obtained. The point is now small: prepare the equipment and take measurements. The equipment required for the experiment is rather modest, especially in comparison with the giant modern particle accelerators. The electron, accelerated to just an energy of $10 \mathrm{Mev}$, has a speed close to $0.99 c$, so the outcome would be worth investment.

\section{Conclusion}

In the paper [9], an example of an irreconcilable contradiction between the special theory of relativity on the one hand and the general theory of relativity on the other, arising in a mechanical system of a cyclic type, is sorted out. The subject of the present work is a strange situation emerged in the doctrine of electromagnetism based on the relativistic approach. First, it is the presence of an implicit axiom about the constant expiration of the potential with the speed of light in an electrostatic field from its source-a point charge. For an accelerated charge, the role of carriers can be assigned to the emitted photons. However, in the case of a charge moving without acceleration, it remains an unsolved mystery: what kind of "superfine matter" constantly transports the potential in an electrostatic field? Secondly, the type of deformation of the Coulomb field of a point charge is observed in uniform motion with a constant velocity $\boldsymbol{v}=$ const . The correct interpretation of the geometry arising in this way leads to the unavoidable inconsistency in the picture of the equipotential surfaces in the electrostatic field of the charged sphere. Third, it is the nature of the movement of static fields when moving their carriers. These psychological invisibilities, surprisingly escaping the attention of physicists for a whole century, caused a distorted view of physical reality, in which there is no room for electro-magneto-kinematics. The absence to this day of experimental confirmation of the deformed configuration of the field of a point charge, moving with a constant velocity $\boldsymbol{v}$, presents a serious challenge for experimenters. 


\section{Acknowledgements}

The author sincerely thanks Mr Michael Kyle for his valuable help while preparing this paper.

\section{Conflicts of Interest}

The author declares no conflicts of interest regarding the publication of this paper.

\section{References}

[1] Heaviside, O. (1888) The Electrician, 22, 147-148.

[2] Leus, V.A. (2013) Progress in Electromagnetics Research M, 32, 27-41. https://doi.org/10.2528/PIERM13043008

[3] Leus, V.A. and Taylor, S. (2018) International Journal of Applied Mathematics and Theoretical Physics, 4, 91-97.

[4] Landau, L.D. and Lifshitz, E.M. (1967) Teorija Polja [Field Theory]. 5th Edition, Nauka, Moscow. (In Russian)

[5] Levich, V.G. (1962) Kurs teoreticheskoj fiziki [Course of Theoretical Physics]. Vol. 1, Fizmatgiz, Moscow. (In Russian)

[6] Leus, V.A. (2018) Science and Engineering Investigations, 7, 30-36. http://www.ijsei.com/papers/ijsei-77918-03.pdf

[7] Edwards, W.F., Kenyon, C.S. and Lemon, D.K. (1976) Physical Review D, 14, 922-938. https://doi.org/10.1103/PhysRevD.14.922

[8] Bonnet, G. (1981) Physics Letters, 82A, 465-467. https://doi.org/10.1016/0375-9601(81)90282-6

[9] Leus, V.A. (2018) Journal of Modern Physics, 9, 1043-1051. https://doi.org/10.4236/jmp.2018.95066 